\title{
Soft x-ray resonant reflectivity of low- $Z$ material thin films
}

\author{
Cheng Wang, T. Araki, and H. Ade ${ }^{\mathrm{a})}$ \\ Department of Physics, North Carolina State University, Raleigh, North Carolina 27695
}

(Received 20 September 2005; accepted 19 October 2005; published online 17 November 2005)

\begin{abstract}
Soft $\mathrm{x}$-ray resonant reflectivity, a method for low- $Z$ materials that combines aspects of neutron reflectivity and $\mathrm{x}$-ray reflectivity, is presented. Resonant reflectivity provides enhanced and selective sensitivity to specific chemical moieties near the absorption edges of constituent elements and was demonstrated through the characterization of a bilayer polymer thin film. The relative reflectivity of a particular interface could be tuned by adjusting the incident photon energy near the carbon 1s absorption edge. The resulting chemical specificity is analogous to using deuteration as a tracer or marker in neutron reflectivity, but without requiring special sample synthesis or preparation. (C) 2005 American Institute of Physics. [DOI: 10.1063/1.2136353]
\end{abstract}

Neutron reflectivity (NR) and x-ray reflectivity (XR) are well established complementary research tools to characterize important parameters of hard- and soft-condensed matter films such as film thickness, interfacial width, diffusion profiles, and surface roughness. ${ }^{1-4}$ Applications range from polymer thin films, to biomembranes, magnetic multilayers and oxide thin films. The relative availability of NR and XR and their respective signal-to-noise $(\mathrm{S} / \mathrm{N})$ ratios, sensitivities, and the scattering vector ranges ( $q$-range) practically achievable have allowed both techniques to prosper. Sensitivity in conventional hard $\mathrm{x}$-ray scattering and reflectivity depends on the relative electron density of constituent material phases. Hence, differences in high atomic number $(Z)$ materials provide better inherent contrast than differences in low $Z$ materials and the characterization of some low $Z$ systems has been difficult. Neutrons have highly variable scattering length densities as a function of $Z$ and even neutron number $N$. For carbonaceous soft-condensed matter, the contrast for neutrons can be enhanced enormously by deuterating one species. ${ }^{5,6}$ On the other hand, NR has some known limitations and the excellent $\mathrm{S} / \mathrm{N}$ ratio and high $q$-range achievable for conventional XR allows the use of Fourier transform methods to characterize, for example, polymer bilayer samples with similar or superior results than achievable with neutrons. ${ }^{7}$

The general principle of resonant $\mathrm{x}$-ray reflectivity (RXR) has been previously recognized. ${ }^{8-11}$ However, with the exception of some magnetic materials characterization (e.g., Refs. 10 and 11), fine structure features of the resonance effects have not been utilized. We extend the resonant principle to the carbon $K$-edge and achieve compositional sensitivity through the use of fine structure features of the complex index of refraction. We show through experiments and simulations that RXR is particularly powerful in characterizing carbonaceous materials containing low $Z$ elements (i.e., carbon, oxygen, nitrogen, etc.). Using photons in the 270-320 eV energy range, measurements were performed on thin film bilayers of polystyrene (PS) on top of poly(methyl methacrylate) (PMMA). Large changes in reflectivity at an interface and the resulting interference in thin films and multilayers are observed as the complex index of refraction, $n=1-\delta-i \beta$, changes rapidly as a function of photon energy.

\footnotetext{
a) Author to whom correspondence should be addressed; electronic mail:
} harald_ade@ncsu.edu
Through the use of specific energies, the sensitivity to the polymer-polymer or polymer-vacuum interface can be selectively enhanced.

The samples were prepared on silicon (100) wafers (Wafer World), which were cleaved into $2.5 \mathrm{~cm} \times 2.5 \mathrm{~cm}$ substrates, cleaned according to a modified Shirake method, ${ }^{12}$ then soaked in aqueous HF $(10 \% \mathrm{v} / \mathrm{v})$ for $\sim 1 \mathrm{~min}$ and rinsed with DI water. PMMA (Scientific Polymer Products, $M_{w}$ $=75 \mathrm{~kg} / \mathrm{mol}, T_{g} \approx 115^{\circ} \mathrm{C}$ ) was spun from Toluene (SigmaAldrich) on top of the substrates, annealed in vacuum at $T$ $=120{ }^{\circ} \mathrm{C}$ for $5 \mathrm{~min}$ and quenched to room temperature. Subsequently, PS (Scientific Polymer Products, $M_{w}=45 \mathrm{~kg} / \mathrm{mol}$, $T_{g} \approx 100{ }^{\circ} \mathrm{C}$ ) was spun from the selective solvent 1-chloropentane (Sigma-Aldrich) directly on top of the PMMA single layer. The bilayers were annealed in vacuum at $120{ }^{\circ} \mathrm{C}$ for $5 \mathrm{~min}$. Near edge $\mathrm{x}$-ray absorption fine structure (NEXAFS) spectra of reference samples were acquired at beamline 5.3.2 at the Advanced Light Source (ALS). ${ }^{13}$ Reflectivity $(\theta-2 \theta)$ data sets were acquired at beamline 6.3.2 at the ALS in a high vacuum $\left(\sim 10^{-7}\right.$ Torr $)$. The data acquisition time was about $10 \mathrm{~min}$ per angular scan $\left(\theta=0-40^{\circ}\right)$. The photon energy was calibrated by measuring absorption spectra of the samples at BL6.3.2 and comparison to known spectra. ${ }^{13}$ To detect and avoid radiation damage, some scans were repeated and the sample was translated occasionally to expose a fresh sample area. Simulations and fits were performed using the noncommercial program IMD. ${ }^{14}$

Figure 1 shows $\delta$ and $\beta$ as a function of energy for PS, PMMA, and poly(2-vinylpyridine) (P2VP). $\beta$ was obtained from transmission NEXAFS spectra and scaling the pre- and post-edge to the Henke atomic scattering database, ${ }^{15}$ taking into account the known composition of these materials. The dispersion $\delta$ was calculated from $\beta$ through Kramers-Kronig integral relations. ${ }^{16}$ Clearly, the complex index of refraction exhibits rapid changes near the absorption edge that depend strongly on composition. Data from P2VP is included to provide a sense on how drastically $\delta$ and $\beta$ can change if just a single carbon atom is replaced by a nitrogen hetero-atom in an aromatic group. In general, the fine structure features in $\beta$ and $\delta$ are much more varied and pronounced in carbonaceous than in magnetic materials. ${ }^{10,11}$ The spectral richness of $\beta$ is already used extensively in NEXAFS spectroscopy and microscopy of carbonaceous materials, ${ }^{13,17-19}$ which can serve as a guide to how powerful and general soft $\mathrm{x}$-ray resonant 


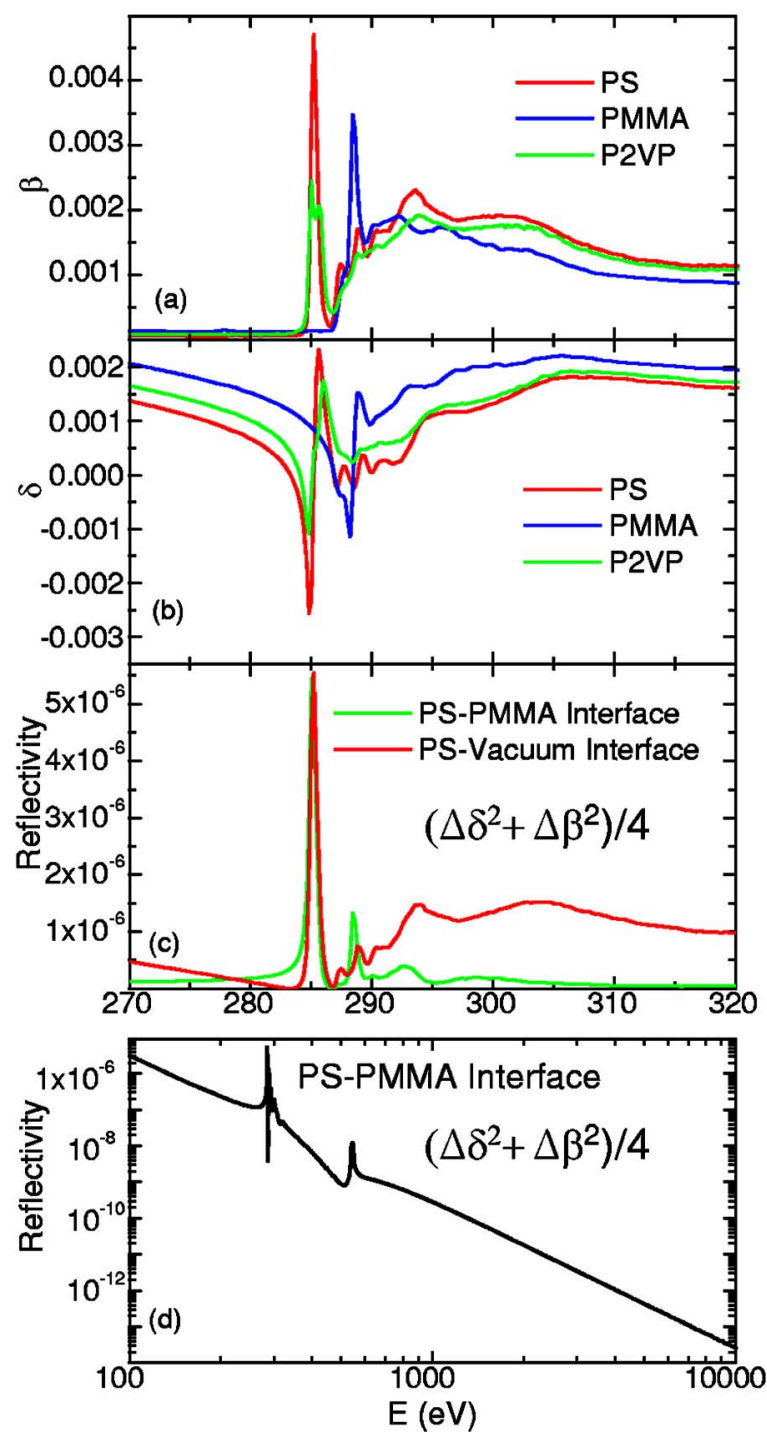

FIG. 1. (Color online) Absorption $\beta$ (a) and dispersion $\delta$ (b) of complex index of refraction for PS, PMMA, and P2VP near the carbon 1-s absorption edge. Both $\delta$ and $\beta$ exhibit rapidly changing fine structure that can be exploited to tune the reflectivity of interfaces. (c) Reflectivity of PS-PMMA and PS-vacuum interfaces near the carbon $K$-edge. (d) Reflectivity of the PS-PMMA interface for a larger range of photon energies. The feature near the oxygen edge at $\sim 540 \mathrm{eV}$ does not take into account the finer details that one would expect for PMMA, but is solely due to the edge jump based on the absorption data tabulated by Henke (Ref. 15).

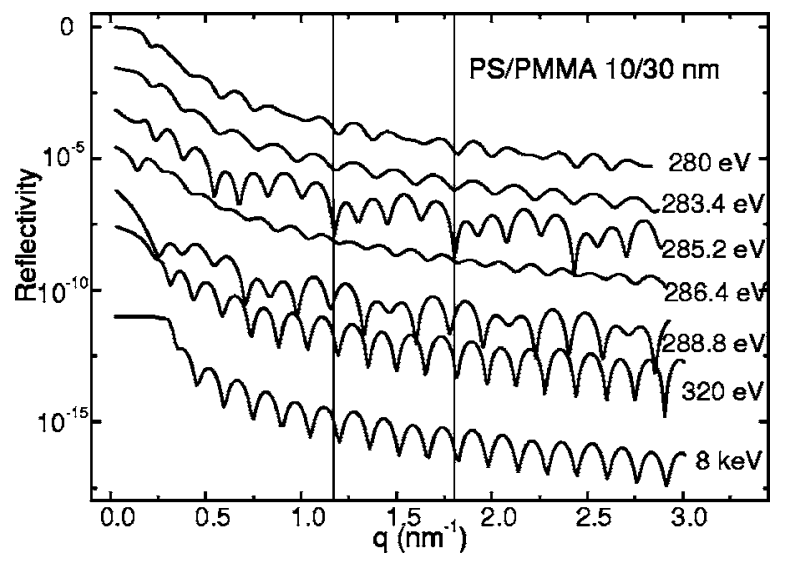

FIG. 2. Calculated reflectivity of a perfectly smooth $10 / 30 \mathrm{~nm}$ PS/PMMA bilayer at selected photon energies, using the values of $\delta$ and $\beta$ as displayed in Fig. 1. At $8 \mathrm{keV}$ and $320 \mathrm{eV}$, the fringes are dominated by sensitivity to the total film thickness. In contrast to this, 280, 283.4, 285.2, and $288.8 \mathrm{eV}$ show strong sensitivity to the polymer-polymer interface.

Down 19 .

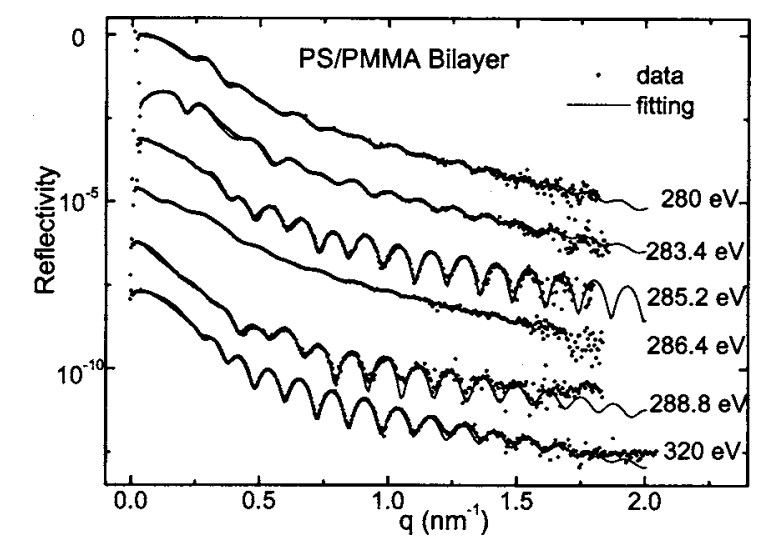

FIG. 3. Comparison of experimental data (dot) with fits (solid line) for a PS/PMMA bilayer. Results of the fits are listed in Table I. Data is from three different sample areas.

reflectivity will be for systems not utilized in the present demonstration.

The reflected intensity of a real sample and the extent of the observed oscillations as a function of scattering vector $q$ that correspond to destructive and constructive interference depend on the relative intensities of the reflection at each interface in a multilayer system as well as the absorption in each layer. The photon energy dependence of this complex behavior is best illustrated by simulations. Simulations of $10 \mathrm{~nm}$ PS on top of $30 \mathrm{~nm}$ PMMA on Silicon show the most salient aspects most clearly. The calculated reflectivity is presented in Fig. 2 for several $x$-ray energies, using the values of $\delta$ and $\beta$ as displayed in Fig. 1. Surface and interface roughness are set to zero. Almost uniform modulations with $\Delta q=0.16 \mathrm{~nm}^{-1}$ are observed for 286.4, 320 and $8000 \mathrm{eV}$. These modulations arise from interference corresponding to the total film thickness and show very limited sensitivity to the polymer-polymer interface. The amplitude of the modulation is very small for $286.4 \mathrm{eV}$ due to the relative low reflectivity of the PS-vacuum and PS-PMMA interface at this energy and largest at $320 \mathrm{eV}$ due to the relatively well matched reflection intensities of the PS/Vacuum and PMMAsubstrate interfaces. In contrast, strong interference effects related to the bilayer structure are readily observed at 280 , 285.2 and $288.8 \mathrm{eV}$. The $285.2 \mathrm{eV}$ simulation has, for example, recognizable strong interference at $\Delta q=0.63 \mathrm{~nm}^{-1}$, i.e., at 4 times the $\Delta q$ that corresponds to the full layer. This directly implies strong sensitivity to the $10 \mathrm{~nm}$ thick PS layer. At $280 \mathrm{eV}$, good sensitivity to the PS-PMMA interface is observed, although the overall modulation is reduced compared to $285.2 \mathrm{eV}$. A potentially interesting energy is $283.4 \mathrm{eV}$, where the refraction index of PS $(\delta=0, \beta \approx 0)$ is very close to the refraction index of vacuum $(\delta=0, \beta=0)$ (Fig. 1). This makes the PS almost invisible at this energy and the reflectivity modulation reflects the information about the PMMA layer. The fringe spacing $\Delta q$ at $320 \mathrm{eV}$ is $3 / 4$ of the fringe spacing at $283.4 \mathrm{eV}$, corresponding to the $30 \mathrm{~nm}$ PMMA instead of the $40 \mathrm{~nm}$ total thickness of the bilayer. These simulations show that by choosing specific photon energies, the sensitivity of RXR to a specific interface should be greatly improved relative to conventional XR.

Figure 3 shows experimental data for a PS/PMMA bilayer for the same photon energies used in the simulations shown in Fig. 2. Overall, similar trends as in the simulations can be found in the experiment and the same strong energy AlP license or copyright; see http://apl.aip.org/apl/copyright.jsp 
TABLE I. Results of fitting the reflectance of the PS/PMMA bilayer. $\delta_{\mathrm{PS}}, \delta_{\mathrm{PMMA}}$, and $\beta_{\mathrm{PS}}, \beta_{\mathrm{PMMA}}$ are the dispersion and the absorption components of the complex index of refraction and $d_{\mathrm{PS}}$ and $d_{\mathrm{PMMA}}$ are the thickness of the PS and PMMA layers, respectively. $\sigma_{\text {sur }}$ and $\sigma_{\text {int }}$ are the surface roughness and interface roughness, respectively.

\begin{tabular}{ccccccccc}
\hline \hline$E(\mathrm{eV})$ & $\delta_{\mathrm{PS}}$ & $\beta_{\mathrm{PS}}$ & $\delta_{\text {PMMA }}$ & $\beta_{\text {PMMA }}$ & $d_{\text {PS }}(\mathrm{nm})$ & $d_{\text {PMMA }}(\mathrm{nm})$ & $\sigma_{\text {sur }}(\mathrm{nm})$ & $\sigma_{\text {int }}(\mathrm{nm})$ \\
\hline 280.0 & $0.00067^{\mathrm{a}}$ & $0.00007^{\mathrm{a}}$ & $0.00152^{\mathrm{a}}$ & $0.00013^{\mathrm{a}}$ & 18.4 & 31.2 & 0.66 \\
284.3 & -0.00070 & 0.00008 & 0.00126 & 0.00015 & 17.6 & 30.6 & 0.72 \\
285.2 & 0.00186 & 0.00378 & 0.00165 & 0.00080 & 17.0 & 30.8 & 0.69 \\
288.8 & -0.00110 & 0.00233 & 0.00092 & 0.00330 & 17.6 & 31.2 & 1.89 \\
320.0 & $0.00161^{\mathrm{a}}$ & $0.00115^{\mathrm{a}}$ & $0.00195^{\mathrm{a}}$ & $0.00088^{\mathrm{a}}$ & 17.7 & 30.9 & 1.92 & 1.18 \\
average & & & & & 17.7 & 30.9 & 0.93 & 2.36 \\
\hline \hline
\end{tabular}

${ }^{\mathrm{a}}$ Values not fit. NEXAFS-derived values from Fig. 1 were utilized.

dependence can be observed. At $286.4 \mathrm{eV}$, limited interference is observed between any interfaces. The data for 280 , 283.4, 285.2, and $288.8 \mathrm{eV}$ shows clear interference and sensitivity to the polymer-polymer interface. The effects are weaker at 285.2 and $280 \mathrm{eV}$ than anticipated. In addition, the experiment at $283.4 \mathrm{eV}$ shows strong PS-PMMA and PSvacuum interface sensitivity whereas the simulation indicated the PS layer should be nearly "invisible." This points to inaccuracies in the values of $\delta$ and $\beta$ as displayed in Fig. 1 , possibly due to differences in resolving power, saturation levels or energy calibration between different beamlines or inaccuracies in Henke tables values. All aspects of the data acquisition can be improved significantly in the future.

Fits of the reflectance at 280 and $320 \mathrm{eV}$ were performed using the values of $\delta$ and $\beta$ shown in Fig. 1. Better fits could be achieved for the other energies ( $\delta$ and $\beta$ oscillate rapidly) if one allowed for simultaneous fitting of the optical constants and other thin film parameters. The data measured at $286.4 \mathrm{eV}$ was not fit, due to lack of sensitivity to all the interfaces at this energy. Fitting results for the other five x-ray energies are quite consistent (see details in Table I). The average PS ad PMMA thickness of the bilayer is 17.7 and $30.9 \mathrm{~nm}$, respectively. The average surface roughness is $0.85 \mathrm{~nm}$ and the average interfacial roughness is $2.0 \mathrm{~nm}$. The change in sample location for different photon energy may account for the relatively larger surface roughness observed at 288.8 and $320 \mathrm{eV}$. In addition, the sensitivity to the polymer-polymer interface is small at $320 \mathrm{eV}$, and the interfacial roughness value derived would be relative uncertain. The interfacial width determined with RXR is virtually identical to that measured previously by $\mathrm{XR}$ and $\mathrm{NR},{ }^{7,20,21}$ yet was achieved without deuteration or special analysis methods.

We have demonstrated that RXR data can be acquired from soft-condensed, carbonaceous materials over a useful $q$ range. More importantly, the strongly oscillatory energy dependence of the reflectivity for the various interfaces provides tunable, high sensitivity to a particular interface. This mimics the contrast enhancement achieved through deuteration used in NR, yet does not require special chemical procedures. Generally, there are no constraints on which low $Z$ system can be studied. In the future, data sets at multiple energies can be fitted simultaneously to provide a unique composition profile of complicated thin film structures. ${ }^{9}$ The combination of selectable inherent chemical sensitivity, achievable $q$-range and $\mathrm{S} / \mathrm{N}$ ratio, and analysis tools available should make soft $\mathrm{x}$-rays resonant reflectivity a powerful complementary characterization tools to NR and conventional XR.

The authors gratefully acknowledge S. Harton for preparing some of the bilayer samples, J. Kortright and G. Mitchell for helpful discussions, and Eric Gullikson for help with some measurements. Funding provided by the U. S. Department of Energy (DE-FG02-98ER45737). Data acquired at beamlines 5.3.2 and 6.3.2 at the ALS, which is supported by the Director of the Office of Science, Department of Energy, under Contract No. DE-AC02-05CH11231.

${ }^{1}$ E. Chason and T. M. Mayer, Crit. Rev. Solid State Mater. Sci. 22, 1 (1997).

${ }^{2}$ M. Stamm and D. W. Schubert, Annu. Rev. Mater. Sci. 25, 295 (1995).

${ }^{3}$ S. K. Sinha, E. B. Sirota, S. Garoff, and H. B. Stanley, Phys. Rev. B 38, 2297 (1988).

${ }^{4}$ X. L. Zhou and S. H. Chen, Phys. Rep. 257, 223 (1995).

${ }^{5}$ S. H. Anastasiadis, T. P. Russell, S. K. Satija, and C. F. Majkrzak, J. Chem. Phys. 92, 5677 (1990).

${ }^{6}$ M. L. Fernandez, J. S. Higgins, J. Penfold, R. C. Ward, C. Shackleton, and D. J. Walsh, Polymer 29, 1923 (1988).

${ }^{7}$ O. H. Seeck, I. D. Kaendler, M. Tolan, M. Shin, M. H. Rafailovich, J. Sokolov, and R. Kolb, Appl. Phys. Lett. 76, 2713 (2000).

${ }^{8}$ J. Bai, E. E. Fullerton, and P. A. Montano, Physica B 221, 411 (1996).

${ }^{9}$ M. K. Sanyal and S. K. Sinha, Europhys. Lett. 21, 691 (1993).

${ }^{10}$ E. Meltchakov, H.-C. Mertins, M. Scheer, S. D. Fonzo, W. Jark, and F. Schäfers, J. Magn. Magn. Mater. 240, 550 (2002).

${ }^{11}$ K. S. Lee, S. K. Kim, and J. B. Kortright, Appl. Phys. Lett. 83, 3764 (2003).

${ }^{12}$ K. Shin, X. Hu, X. Zheng, M. H. Rafailovich, J. Sokolov, V. Zaitsev, and S. A. Schwarz, Macromolecules 34, 4993 (2001).

${ }^{13}$ O. Dhez, H. Ade, and S. Urquhart, J. Electron Spectrosc. Relat. Phenom. 128, 85 (2003).

${ }^{14}$ D. L. Windt, Comput. Phys. 12, 360 (1998).

${ }^{15}$ B. L. Henke, E. M. Gullikson, and J. C. Davis, At. Data Nucl. Data Tables 54, 181 (1993).

${ }^{16} \mathrm{D}$. Attwood, Soft X-rays And Extreme Ultraviolet Radiation, Principles and Applications (Cambridge University Press, Cambridge, 2000).

${ }^{17}$ J. Stöhr, NEXAFS Spectroscopy (Springer, New York, 1992).

${ }^{18}$ H. Ade, X. Zhang, S. Cameron, C. Costello, J. Kirz, and S. Williams, Science 258, 972 (1992).

${ }^{19}$ H. Ade, Trends Polym. Sci. 5, 58 (1997).

${ }^{20}$ M. Sferrazza, C. Xiao, R. A. L. Jones, D. G. Bucknall, J. Webster, and J. Penfold, Phys. Rev. Lett. 78, 3693 (1997).

${ }^{21}$ D. W. Schubert and M. Stamm, Europhys. Lett. 35, 419 (1996). 